\title{
Iterative Methods for Nonconvex Equilibrium Problems in Uniformly Convex and Uniformly Smooth Banach Spaces
}

\author{
Messaoud Bounkhel \\ Department of Mathematics, College of Science, P.O. Box 2455, Riyadh 11451, Saudi Arabia \\ Correspondence should be addressed to Messaoud Bounkhel; bounkhel@ksu.edu.sa \\ Received 8 October 2014; Accepted 19 February 2015 \\ Academic Editor: P. Veeramani
}

Copyright (C) 2015 Messaoud Bounkhel. This is an open access article distributed under the Creative Commons Attribution License, which permits unrestricted use, distribution, and reproduction in any medium, provided the original work is properly cited.

We suggest and study the convergence of some new iterative schemes for solving nonconvex equilibrium problems in Banach spaces. Many existing results have been obtained as particular cases.

\section{Introduction}

Let $X$ be a Banach space, and let $X^{*}$ be the dual space of $X$. Let $\langle\cdot, \cdot\rangle$ denote the duality pairing of $X^{*}$ and $X$. Let $C$ be a nonempty closed subset of $X$ and let $F: X \times X \rightarrow \mathbb{R}$ be a bifunction satisfying $F(x, x)=0$ for all $x \in C$. A prototype of equilibrium problem associated with a closed convex set $C$ and a convex bifunction $F$ is given as follows:

Find $\bar{x} \in C$ such that

$$
F(\bar{x}, x) \geq 0 \quad \forall x \in C .
$$

In this paper we introduce and study two appropriate extensions of $(E P(C, F))$ from the convex case to the nonconvex case in Banach spaces setting. We consider the two following generalized equilibrium problems associated with $C, \rho$, and $F$ (resp., denoted by $(G E P 1(C, \rho, F))$ and $(G E P 2(C, \rho, F)))$ :

Find $\bar{x} \in C$ such that

$$
F(\bar{x}, x)+\rho V(J(\bar{x}), x) \geq 0 \quad \forall x \in C,
$$$$
(G E P 1(C, \rho, F))
$$

Find $\bar{x} \in C$ such that

$$
F(\bar{x}, x)+\rho V(J(x), \bar{x}) \geq 0 \quad \forall x \in C,
$$$$
(G E P 2(C, \rho, F))
$$

where $J: X \rightarrow X^{*}$ is the normalized duality mapping and $V: X^{*} \times X \rightarrow \mathbb{R}$ is the functional defined by

$$
V\left(x^{*}, x\right)=\left\|x^{*}\right\|^{2}-2\left\langle x^{*}, x\right\rangle+\|x\|^{2} .
$$

Due to the nonsymmetry of the terms $V(J(x), \bar{x})$ and $V(J(\bar{x}), x)$ we can think about the symmetric functional $W(x, \bar{x}):=(1 / 2)[V(J(\bar{x}), x)+V(J(x), \bar{x})]$. Thus, we can consider one more appropriate extension of equilibrium problem from Hilbert spaces to Banach spaces as follows:

$$
\begin{aligned}
& \text { Find } \bar{x} \in C \text { such that } \\
& F(\bar{x}, x)+\rho W(\bar{x}, x) \geq 0 \quad \forall x \in C . \quad(G E P 3(C, \rho, F))
\end{aligned}
$$

The reason for saying that the above three generalized equilibrium problems are the appropriate extensions from Hilbert spaces to Banach spaces and from convex cases to nonconvex cases is that in Hilbert spaces we have $W(\bar{x}, x)=V(J(x), \bar{x})=$ $V(J(\bar{x}), x)=\|x-\bar{x}\|^{2}$ and the convex case is covered by taking $\rho=0$. Obviously, any solution of $(G E P 1(C, \rho, F))$ and $(G E P 2(C, \rho, F))$ is a solution of $(G E P 3(C, \rho, F))$. Thus, we are going to study the convergence of new iterative schemes to solutions of $(G E P 1(C, \rho, F))$ and $(G E P 2(C, \rho, F))$.

We list some important properties of $V$ needed in our proofs, when $X$ is a reflexive smooth Banach space:

(i) $V\left(x^{*}, x\right) \geq 0$, for all $x \in X, x^{*} \in X^{*}$;

(ii) $\left(\left\|x^{*}\right\|-\|x\|\right)^{2} \leq V\left(x^{*}, x\right) \leq\left(\left\|x^{*}\right\|+\|x\|\right)^{2}$, for all $x \in X$, $x^{*} \in X^{*}$;

(iii) $V(J(x), x)=0$, for all $x \in X$;

(iv) $V\left(x^{*}, x\right)$ is continuous and $V$ is convex with respect to $x$ when $x^{*}$ is fixed and convex with respect to $x^{*}$ when $x$ is fixed; 
(v) whenever the space $X$ is smooth, the functional $V\left(x^{*}, \cdot\right)$ is differentiable and $\operatorname{grad}_{x} V\left(x^{*}, \cdot\right)(\bar{x})=$ $2\left(J(\bar{x})-x^{*}\right)$;

(vi) $V\left(x^{*}, x\right)=0$ if and only if $x^{*}=J(x)$.

The proposed generalized equilibrium problems extend many existing equilibrium problems and variational inequalities from the convex case to the nonconvex case and from Hilbert spaces setting to Banach spaces setting.

(1) If $X$ is a Hilbert space, the duality mapping is the identity operator and $W(\bar{x}, x)=V(J(\bar{x}), x)=V(J(\bar{x})$, $x)=\|x-\bar{x}\|^{2}$ and so $(G E P 1(C, \rho, F)),(G E P 2(C, \rho, F))$, and $(G E P 3(C, \rho, F))$ become as follows:

Find $\bar{x} \in C$ such that

$$
F(\bar{x}, x)+\rho\|x-\bar{x}\|^{2} \geq 0 \quad \forall x \in C,
$$

which has been introduced and studied in [1]. The same problem has been studied by Noor [2] and many authors (see, e.g., [3-5]).

(2) If $X$ is a Hilbert space, $C$ is a convex closed set in $X, F$ is a convex bifunction, and $\rho=0$, all the generalized equilibrium problems $(G E P 1(C, \rho, F))$, $(G E P 2(C, \rho, F))$, and $(G E P 3(C, \rho, F))$ become as follows:

$$
\begin{aligned}
& \text { Find } \bar{x} \in C \text { such that } \\
& F(\bar{x}, x) \geq 0 \quad \forall x \in C,
\end{aligned}
$$

which has been studied in various works (see, e.g., [3, 4] and the references therein).

(3) If $F(x, y)=\langle T(x), y-x\rangle$, with $T: X \rightarrow X^{*}$ being a nonlinear operator, then $(G E P 1(C, \rho, F))$ and $(G E P 2(C, \rho, F))$ reduce, respectively, to the following:

Find $\bar{x} \in C$ such that

$$
\langle T(\bar{x}), x-\bar{x}\rangle+\rho V(J(\bar{x}), x) \geq 0 \quad \forall x \in C,
$$

Find $\bar{x} \in C$ such that

$$
\langle T(\bar{x}), x-\bar{x}\rangle+\rho V(J(x), \bar{x}) \geq 0 \quad \forall x \in C .
$$

These inequalities are new even in Banach spaces. However, it has been studied, in Hilbert spaces, in [6], when $C$ is a uniformly prox-regular set (which is not necessarily a convex set) (see also $[1,2,4]$ ). By taking $\rho=0$ the last inequality becomes as follows:

Find $\bar{x} \in C$ such that

$$
\langle T(\bar{x}), x-\bar{x}\rangle \geq 0 \quad \forall x \in C,
$$

which is known as the classical variational inequality introduced and studied by Stampacchia in [7].

Our main aim of the present paper is to suggest and analyze some iterative schemes for solving the proposed generalized equilibrium problems $(G E P 1(C, \rho, F))$ and $(G E P 2(C, \rho, F))$.

\section{Main Results}

We recall some definitions and results on $p$-uniformly convex and $q$-uniformly smooth Banach spaces (see, e.g., $[8,9]$ ). The moduli of convexity and smoothness of $X$ are defined, respectively, by

$$
\begin{array}{r}
\delta_{X}(\epsilon)=\inf \left\{1-\left\|\frac{x+y}{2}\right\|:\|x\|=\|y\|=1,\|x-y\|=\epsilon\right\}, \\
0 \leq \epsilon \leq 2, \\
\rho_{X}(t)=\sup \left\{\frac{1}{2}(\|x+y\|+\|x-y\|)-1:\|x\|=1,\|y\|=t\right\},
\end{array}
$$

The space $X$ is said to be uniformly convex whenever $\delta_{X}(\epsilon)>$ 0 for all $0<\epsilon \leq 2$ and is said to be uniformly smooth whenever $\lim _{t \downarrow 0} \rho_{X}(t)=0$. Let $p, q>1$ be real numbers. The space $X$ is said to be $p$-uniformly convex (resp., $q$-uniformly smooth) if there is a constant $c>0$ such that

$$
\delta_{X}(\epsilon) \geq c \epsilon^{p} \quad\left(\text { resp. } \rho_{X}(t) \leq c t^{q}\right)
$$

It is known (see, e.g., $[8,9]$ ) that uniformly convex Banach spaces are reflexive, strictly convex and that uniformly smooth Banach spaces are reflexive. If $X$ is a $p$-uniformly convex Banach space, then $X^{*}$ is a $p^{\prime}$-uniformly smooth Banach space, where $p^{\prime}=p /(p-1)$ is the conjugate number of $p$. If $X$ is a $q$-uniformly smooth Banach space, then $X^{*}$ is a $q^{\prime}$-uniformly convex Banach space, where $q^{\prime}=q /(q-1)$.

The following lemma is needed in our proofs and for its proof we refer to [10].

Lemma 1. Let $p, q>1, E$ be a $p$-uniformly convex and $q$ uniformly smooth Banach space, and let $S$ be a bounded set. Then there exist constants $\eta, \kappa>0$ such that

$$
\eta^{-1}\|x-y\|^{p} \leq V(J(x), y) \leq \kappa^{-1}\|x-y\|^{q}, \quad \forall x, y \in S .
$$

Let $X$ be a reflexive smooth Banach space and let $f: X \rightarrow$ $\mathbb{R} \cup\{+\infty\}$ be a function and $x \in X$, where $f$ is finite. We recall from [10] that the proximal subdifferential $\partial^{\pi} f(x)$ is the set of all $x^{*} \in X^{*}$ for which there are $\sigma>0$ and $\delta>0$ so that

$$
\left\langle x^{*}, x^{\prime}-x\right\rangle \leq f\left(x^{\prime}\right)-f(x)+\sigma V\left(J(x), x^{\prime}\right),
$$

for all $x^{\prime}$ around $x$. We also recall ([10]) that the proximal normal cone of a nonempty closed subset $S$ in $X$ at $x \in S$ is defined by $N^{\pi}(S ; x)=\partial^{\pi} \psi_{S}(x)$, where $\psi_{S}$ is the indicator function of $S$. The following proposition summarizes some properties of $\partial^{\pi} f(x)$ and $N^{\pi}(S, x)$ that we need in our proofs. For their proofs we refer the reader to [10].

Proposition 2. Let $X$ be a reflexive smooth Banach space. Then the following assertions hold: 
(1) if $f$ is l.s.c. convex, then $\partial^{\pi} f(x)$ coincides with the subdifferential $\partial^{\text {Conv. }} f(x)$ in the sense of convex analysis defined by

$$
\begin{aligned}
\partial^{\text {Conv. }} f(x) & \\
=\left\{x^{*} \in X^{*}:\right. & \left\langle x^{*}, x^{\prime}-x\right\rangle \\
& \left.\leq f\left(x^{\prime}\right)-f(x), \forall x \in \operatorname{dom} f\right\} ;
\end{aligned}
$$

(2) if $S$ is a closed convex set, then $N^{\pi}(S ; x)$ coincides with the normal cone $N^{\text {Conv. }}(S ; x)$ in the sense of convex analysis defined by

$N^{\text {Conv. }}(S ; x)=\left\{x^{*} \in X^{*}:\left\langle x^{*}, x^{\prime}-x\right\rangle \leq 0, \forall x \in S\right\}$

(3) if $\bar{x}$ is a local minimum of $f$, then $0 \in \partial^{\pi} f(\bar{x})$;

(4) $\partial^{\pi} d_{S}(x) \subset N^{\pi}(S ; x)$, for any $x \in S$, where $d_{S}$ is the distance function associated with a nonempty closed set $S$ in $X$;

(5) if $f$ is locally Lipschitz around $\bar{x}$ with ratio $K$, then $\partial^{\pi} f(\bar{x}) \subset K \mathbb{B} ;$

(6) $\partial^{\pi} f_{1}(x)+\partial^{\pi} f_{2}(x) \subset \partial^{\pi}\left(f_{1}+f_{2}\right)(x)$;

(7) $N^{\pi}(S ; x)$ is also characterized by the following global inequality:

$$
\begin{array}{r}
x^{*} \in N^{\pi}(S ; x) \Longleftrightarrow\left\langle x^{*}, x^{\prime}-x\right\rangle \leq \sigma V\left(J(x), x^{\prime}\right), \\
\forall x^{\prime} \in S .
\end{array}
$$

We propose the following two iterative schemes:

Select $x_{k+1} \in C$ such that $J\left(x_{k+1}\right) \in J\left(x_{k}\right)+M \lambda_{k} \mathbb{B}$,

$$
\frac{J\left(x_{k}\right)-J\left(x_{k+1}\right)}{\lambda_{k}} \in \partial^{\pi} F\left(x_{k+1}, \cdot\right)\left(x_{k+1}\right)+N^{\pi}\left(C ; x_{k+1}\right) \text {, }
$$

Select $x_{k+1} \in C$ such that $J\left(x_{k+1}\right) \in J\left(x_{k}\right)+M \lambda_{k} \mathbb{B}$,

$$
\frac{J\left(x_{k+1}\right)-J\left(x_{k}\right)}{\lambda_{k}} \in \partial^{\pi} F\left(x_{k}, \cdot\right)\left(x_{k}\right)+N^{\pi}\left(C ; x_{k}\right),
$$

where $M>0$ is a given positive number. Under natural assumptions, we will prove the convergence of a subsequence of the sequence $\left\{x_{k}\right\}$ generated by (GSP1) (resp., (GSP2)) to a solution of $(G E P 1(C, \rho, F))$ (resp., $(G E P 2(C, \rho, F)))$.

To start our study we define two new classes of nonconvex sets and nonconvex functions as follows.

Definition 3. Let $X$ be a reflexive smooth Banach space. For a given $r \in(0, \infty)$, a subset $C$ is $V$-uniformly prox-regular with respect to $r$ provided that for all $x \in C$ and all nonzero $x^{*} \in N^{\pi}(C ; x)$ we have

$$
\left\langle\frac{x^{*}}{\left\|x^{*}\right\|}, x^{\prime}-x\right\rangle \leq \frac{1}{2 r} V\left(J(x), x^{\prime}\right), \quad \forall x^{\prime} \in C .
$$

We use the convention $1 / r=0$ for $r=+\infty$.
Obviously, this class extends the class of uniformly proxregular sets $([11,12])$ from Hilbert spaces to Banach spaces since in Hilbert spaces we have $V\left(J(x), x^{\prime}\right)=\left\|x-x^{\prime}\right\|^{2}$ and the generalized proximal normal cone $N^{\pi}(C ; x)$ coincides with the usual proximal subdifferential $N^{p}(C ; x)$ (see [10] for more details on $N^{\pi}(S ; x)$ and $\left.\partial^{\pi} f(x)\right)$. We point out that a different extension of uniformly prox-regular sets to Banach spaces has been considered and studied recently in [13].

Definition 4. Let $X$ be a reflexive smooth Banach space. Let $f: X \rightarrow \mathbb{R} \cup\{+\infty\}$ be a l.s.c. function and let $S \subset \operatorname{dom} f:=$ $\{x \in X: f(x)<\infty\}$ be a nonempty closed set in $X$. We will say that $f$ is $V$-uniformly prox-regular over $S$ provided that for all $x \in S$ and all $x^{*} \in \partial^{\pi} f(x)$ we have

$$
\begin{array}{r}
\left\langle x^{*}, x^{\prime}-x\right\rangle \leq f\left(x^{\prime}\right)-f(x)+\frac{1}{2 r} V\left(J(x), x^{\prime}\right), \\
\forall x^{\prime} \in S .
\end{array}
$$

We say that $f$ is $V$-prox-regular around $\bar{x} \in \operatorname{dom} f$ provided that $f$ is $V$-uniformly prox-regular over some closed neighborhood of $\bar{x}$; that is, there exists a closed neighborhood $V_{\bar{x}}$ of $\bar{x}$ such that for all $x \in V_{\bar{x}}$, for all $x^{*} \in \partial^{\pi} f(x)$, inequality (14) holds for any $x^{\prime} \in V_{\bar{x}}$.

Example 5. Consider the following.

(1) Any l.s.c. proper convex function is $V$-uniformly prox-regular over any nonempty closed set $S$ in its domain with $r=+\infty$.

(2) The indicator function $\psi_{C}$ of $V$-uniformly proxregular set $C$ is $V$-uniformly prox-regular over $C$ with respect to the same constant $r$.

(3) The distance function $d_{C}$ associated with a $V$ uniformly prox-regular set $C$ is $V$-uniformly proxregular over $C$ with respect to the same constant $r$. Indeed, for any $x \in C$ and for all $x^{*} \in \partial^{\pi} d_{C}(x)$ we have by Parts (4-5) in Proposition 2 that $x^{*} \in$ $N^{\pi}(C ; x)$ with $\left\|x^{*}\right\| \leq 1$ and so by Definition 3 :

$$
\begin{aligned}
\left\langle x^{*}, x^{\prime}-x\right\rangle & \leq \frac{\left\|x^{*}\right\|}{2 r} V\left(J(x), x^{\prime}\right) \\
& \leq \frac{1}{2 r} V\left(J(x), x^{\prime}\right), \quad \forall x^{\prime} \in C,
\end{aligned}
$$

and hence

$$
\begin{aligned}
& \left\langle x^{*}, x^{\prime}-x\right\rangle \\
& \quad \leq d_{C}\left(x^{\prime}\right)-d_{C}(x)+\frac{1}{2 r} V\left(J(x), x^{\prime}\right), \quad \forall x^{\prime} \in C,
\end{aligned}
$$

that is, $d_{C}$ is $V$-uniformly prox-regular over $C$ with respect to the same constant $r$.

(4) Any lower- $C^{2}$ function $f$ over convex strongly compact $K$ in $X$ is $V$-uniformly prox-regular over $K$ with some $r \in(0,+\infty]$ as the next propositions (Propositions 7 and 8) show. 
Definition 6. Let $X$ be a reflexive smooth Banach space. A function $f: X \rightarrow \mathbb{R}$ is said to be lower- $C^{2}$ around a point $\bar{x} \in \operatorname{dom} f$ if there exists an open convex neighborhood $O$ and $\rho \geq 0$ such that $f+(\rho / 2)\|\cdot\|^{2}$ is finite and convex over $O$. We will say that $f$ is lower- $C^{2}$ around a subset $S \subset H$ if it is lower- $C^{2}$ around each point of $S$.

Proposition 7. Let $X$ be a reflexive smooth Banach space and let $K$ be a convex strongly compact set in $X$. If $f$ is a lower- $C^{2}$ function on $K$, then $f$ is uniformly lower- $C^{2}$ over $K$; that is, for some $\rho \geq 0$ the function $f+(\rho / 2)\|\cdot\|^{2}$ is finite convex over $K$.

Proof. Assume that $f$ is lower- $C^{2}$ on $K$. For every $x \in K$ there exist, by Definition 6 , an open convex neighborhood $O_{x}$ of $x$ and $\rho_{x} \geq 0$ such that $f+\left(\rho_{x} / 2\right)\|\cdot\|^{2}$ is finite convex over $O_{x}$. The family of open sets $\left\{O_{x}\right\}_{x \in K}$ covers $K$, so by the strong compactness of $K$ there exist points $x_{1}, x_{2}, \ldots, x_{n}$ in $K$ such that $K \subset \bigcup_{k=1}^{k=n} O_{x_{k}}$, and $\rho>0$ sufficiently large such that $f+(\rho / 2)\|\cdot\|^{2}$ is finite convex over $\bigcup_{k=1}^{k=n} O_{x_{k}}$ and so over the convex set $K$. This ensures the uniform lower- $C^{2}$ property of $f$ over $K$ and hence the proof is complete.

The next proposition proves the relationship between uniform lower- $C^{2}$ functions and $V$-uniformly prox-regular functions.

Proposition 8. Let $X$ be a reflexive smooth Banach space, $\rho \geq$ 0 , let $K$ be a convex strongly compact subset of $X$, and let $f$ be a l.s.c function on $K$. If $f$ is uniformly lower- $C^{2}$ on $K$ with some ratio $\rho \geq 0$, then $f$ is $V$-uniformly prox-regular over $K$ with respect to the constant $r=1 / \rho$.

Proof. Assume that $f$ is uniformly lower- $C^{2}$ on $K$ with some ratio $\rho \geq 0$; that is, $f+(\rho / 2)\|\cdot\|^{2}$ is convex over $K$. Let $\bar{x} \in K$ and $x^{*} \in \partial^{\pi} f(\bar{x})$. Then by Part 6 in Proposition 2 we obtain

$$
\begin{aligned}
x^{*}+\rho J(\bar{x}) & \in \partial^{\pi} f(\bar{x})+\operatorname{grad}_{x}\left(\frac{\rho}{2}\|\cdot\|^{2}\right)(\bar{x}) \\
& \subset \partial^{\pi}\left(f+\frac{\rho}{2}\|\cdot\|^{2}\right)(\bar{x}) .
\end{aligned}
$$

Since $f+(\rho / 2)\|\cdot\|^{2}$ is convex over $K$ we have by Part 1 in Proposition 2 that $\partial^{\pi}\left(f+(\rho / 2)\|\cdot\|^{2}\right)(\bar{x})=\partial^{\text {Conv. }}(f+(\rho / 2)$ $\left.\|\cdot\|^{2}\right)(\bar{x})$ and so by the definition of the convex subdifferential:

$$
\begin{aligned}
& \left\langle x^{*}+\rho J(\bar{x}), x-\bar{x}\right\rangle \\
& \quad \leq\left(f+\frac{\rho}{2}\|\cdot\|^{2}\right)(x)-\left(f+\frac{\rho}{2}\|\cdot\|^{2}\right)(\bar{x}), \quad \forall x \in K .
\end{aligned}
$$

This ensures

$$
\begin{aligned}
\left\langle x^{*}, x-\bar{x}\right\rangle \leq & f(x)-f(\bar{x}) \\
& +\frac{\rho}{2}\left[\|x\|^{2}-\|\bar{x}\|^{2}-2\langle J(\bar{x}), x-\bar{x}\rangle\right] \\
\leq & f(x)-f(\bar{x})+\frac{\rho}{2}\left[\|x\|^{2}+\|\bar{x}\|^{2}-2\langle J(\bar{x}), x\rangle\right] \\
\leq & f(x)-f(\bar{x})+\frac{\rho}{2} V(J(\bar{x}), x),
\end{aligned}
$$

for all $x \in K$ and then the function $f$ is $V$-uniformly proxregular over $K$ with constant $r=1 / \rho$ and hence the proof is complete.

Now, we are in position to state and prove our first proposition which extends the main result in [1] from Hilbert spaces to Banach spaces.

Proposition 9. Let $X$ be a reflexive smooth Banach space. IfC is a closed convex set and for all $x \in C$ the function $F(x, \cdot)$ is convex Lipschitz with ratio $M>0$ over an open set containing $C$, then (GSP1) is equivalent to the following subproblem:

Select $x_{k+1} \in C$ such that

$F\left(x_{k+1}, x\right)+\lambda_{k}^{-1}\left\langle J\left(x_{k+1}\right)-J\left(x_{k}\right), x-x_{k+1}\right\rangle \geq 0 \quad \forall x \in C$.

Proof. Let $\left\{x_{k}\right\}_{k} \in C$ be generated by (GSP1), that is,

$$
x_{k+1}^{*} \in \partial^{\pi} F\left(x_{k+1}, \cdot\right)\left(x_{k+1}\right)+N^{\pi}\left(C ; x_{k+1}\right),
$$

with $x_{k+1}^{*}:=\lambda_{k}^{-1}\left[J\left(x_{k}\right)-J\left(x_{k+1}\right)\right]$. Then there exists $y_{k+1}^{*} \in$ $N^{\pi}\left(C ; x_{k+1}\right)$ such that

$$
x_{k+1}^{*}-y_{k+1}^{*} \in \partial^{\pi} F\left(x_{k+1}, \cdot\right)\left(x_{k+1}\right) .
$$

By the convexity of $F\left(x_{k+1}, \cdot\right)$ and Part 1 in Proposition 2 we have $\partial^{\pi} F\left(x_{k+1}, \cdot\right)\left(x_{k+1}\right)=\partial F\left(x_{k+1}, \cdot\right)\left(x_{k+1}\right)$ and so by the definition of the subdifferential for convex functions we have

$$
\begin{aligned}
& \left\langle x_{k+1}^{*}-y_{k+1}^{*}, x-x_{k+1}\right\rangle \\
& \quad \leq F\left(x_{k+1}, x\right)-F\left(x_{k+1}, x_{k+1}\right) \quad \forall x \in C .
\end{aligned}
$$

Since $F\left(x_{k+1}, x_{k+1}\right)=0$, we obtain

$$
\left\langle x_{k+1}^{*}, x-x_{k+1}\right\rangle \leq F\left(x_{k+1}, x\right)+\left\langle y_{k+1}^{*}, x-x_{k+1}\right\rangle \quad \forall x \in C .
$$

On the other hand, by the convexity of $C$ and Part 2 in Proposition 2 we have $N^{\pi}\left(C ; x_{k+1}\right)=N\left(C ; x_{k+1}\right)$ which yields by the definition of convex normal cones the following inequality:

$$
\left\langle y_{k+1}^{*}, x-x_{k+1}\right\rangle \leq 0 \quad \forall x \in C .
$$

Combining this inequality with (23) we obtain

$F\left(x_{k+1}, x\right)+\lambda_{k}^{-1}\left\langle J\left(x_{k+1}\right)-J\left(x_{k}\right), x-x_{k+1}\right\rangle \geq 0 \quad \forall x \in C$.

Conversely, assume that $\left\{x_{k}\right\}_{k}$ is generated by (SP1), that is,

$F\left(x_{k+1}, x\right)+\lambda_{k}^{-1}\left\langle J\left(x_{k+1}\right)-J\left(x_{k}\right), x-x_{k+1}\right\rangle \geq 0 \quad \forall x \in C$.

Let $x_{k+1}^{*}:=\lambda_{k}^{-1}\left[J\left(x_{k}\right)-J\left(x_{k+1}\right)\right]$ and $h(x):=F\left(x_{k+1}, x\right)+$ $\left\langle x_{k+1}^{*}, x_{k+1}-x\right\rangle$. Then the last inequality yields

$$
h(x) \geq h\left(x_{k+1}\right) \quad \forall x \in C .
$$


This means that $x_{k+1}$ is a minimum of $h$ over $C$. Thus, by Part 3 in Proposition 2, we have $0 \in \partial^{\pi}\left(h+\psi_{C}\right)\left(x_{k+1}\right)$ and consequently by the convexity of the set $C$ and the convexity of the function $F\left(x_{k+1}, \cdot\right)$ we can write

$$
\begin{aligned}
0 & \in \partial^{\pi}\left(h+\psi_{C}\right)\left(x_{k+1}\right) \\
& =\partial^{\text {Conv. }}\left(h+\psi_{C}\right)\left(x_{k+1}\right) \\
& =\partial^{\text {Conv. }} h\left(x_{k+1}\right)+N^{\text {Conv. }}\left(C ; x_{k+1}\right) \\
& =\partial^{\text {Conv. }} F\left(x_{k+1}, \cdot\right)\left(x_{k+1}\right)-x_{k+1}^{*}+N^{\text {Conv. }}\left(C ; x_{k+1}\right),
\end{aligned}
$$

and so

$$
x_{k+1}^{*} \in \partial^{\text {Conv. }} F\left(x_{k+1}, \cdot\right)\left(x_{k+1}\right)+N^{\text {Conv. }}\left(C ; x_{k+1}\right) .
$$

On the other hand, since $F\left(x_{k+1}, \cdot\right)$ is Lipschitz continuous with ratio $M$ over an open set containing $C$ we have for all $x, y \in C+\epsilon \mathbb{B}$ for $\epsilon>0$ small enough

$$
\left|F\left(x_{k+1}, x\right)-F\left(x_{k+1}, y\right)\right| \leq M\|x-y\| .
$$

Let $b \in B$. Then, taking $y=x_{k+1}$ and $x:=x_{k+1}+\epsilon b$ in the last inequality yields

$$
\left|F\left(x_{k+1}, x\right)\right| \leq M\left\|x-x_{k+1}\right\|=M \epsilon\|b\| \leq M \epsilon,
$$

and so by $(S P 1)$ we have

$$
\left\langle x_{k+1}^{*}, \epsilon b\right\rangle=\left\langle x_{k+1}^{*}, x-x_{k+1}\right\rangle \leq F\left(x_{k+1}, x\right) \leq M \epsilon,
$$

and hence $\left\langle x_{k+1}^{*}, b\right\rangle \leq M$, for all $b \in \mathbb{B}$, which ensures that $\left\|x_{k+1}^{*}\right\| \leq M$, that is, $J\left(x_{k+1}\right) \in J\left(x_{k}\right)+\lambda_{k} M \mathbb{B}$. Therefore, $\left\{x_{k}\right\}_{k}$ is generated by (GSP1) and the proof is complete.

The following proposition establishes an analogue result for (GSP2) and its proof follows the same lines of the previous proposition. So, its proof is omitted.

Proposition 10. Let $X$ be a reflexive smooth Banach space. If $C$ is a closed convex set and for all $x \in C$ the function $F(x, \cdot)$ is convex Lipschitz with ratio $M>0$ over an open set containing $C$, then (GSP2) is equivalent to the following subproblem:

Select $x_{k+1} \in C$ such that

$$
F\left(x_{k}, x\right)+\lambda_{k}^{-1}\left\langle J\left(x_{k}\right)-J\left(x_{k+1}\right), x-x_{k}\right\rangle \geq 0 \quad \forall x \in C .
$$

The following proposition established a key tool of the proof of our main convergence result of the generalized equilibrium problem $(G E P 1(C, \rho, F))$ in the prox-regular setting.

Proposition 11. Let $X$ be a reflexive smooth Banach space and let $r, r^{\prime} \in(0,+\infty]$. If $C$ is $V$-uniformly $r$-prox-regular and $F\left(x_{k+1}, \cdot\right)$ is $\gamma$-Lipschitz and $V$-uniformly prox-regular over $C$ with ratio $r^{\prime}$, then the sequence $\left\{x_{k}\right\}_{k}$ generated by (GSP1) satisfies the following inequality:

$$
\begin{aligned}
& \lambda_{k}^{-1}\left\langle J\left(x_{k}\right)-J\left(x_{k+1}\right), x-x_{k+1}\right\rangle \\
& \quad \leq F\left(x_{k+1}, x\right)+\left(\frac{\gamma+M+1}{2 \bar{r}}\right) V\left(J\left(x_{k+1}\right), x\right), \quad \forall x \in C,
\end{aligned}
$$

where $\bar{r}=\min \left\{r, r^{\prime}\right\}$.
Proof. Let $\left\{x_{k}\right\}_{k} \subset C$ be generated by (GSP1), that is,

$$
x_{k+1}^{*} \in \partial^{\pi} F\left(x_{k+1}, \cdot\right)\left(x_{k+1}\right)+N^{\pi}\left(C ; x_{k+1}\right), \quad\left\|x_{k+1}^{*}\right\| \leq M,
$$

with $x_{k+1}^{*}:=\left(J\left(x_{k}\right)-J\left(x_{k+1}\right)\right) / \lambda_{k}$. Then there exists $y_{k+1}^{*} \epsilon$ $\partial^{\pi} F\left(x_{k+1}, \cdot\right)\left(x_{k+1}\right)$ such that

$$
x_{k+1}^{*}-y_{k+1}^{*} \in N^{\pi}\left(C ; x_{k+1}\right) .
$$

Since $F\left(x_{k+1}, \cdot\right)$ is $\gamma$-Lipschitz, then by Part 5 in Proposition 2 we have $\left\|y_{k+1}^{*}\right\| \leq \gamma$ and hence $\left\|x_{k+1}^{*}-y_{k+1}^{*}\right\| \leq M+\gamma$. By definition of $V$-uniform prox-regularity of $C$ we have

$$
\begin{array}{r}
\left\langle x_{k+1}^{*}-y_{k+1}^{*}, x-x_{k+1}\right\rangle \leq \frac{\left\|x_{k+1}^{*}-y_{k+1}^{*}\right\|}{2 r} V\left(J\left(x_{k+1}\right), x\right), \\
\forall x \in C,
\end{array}
$$

and hence

$$
\left\langle x_{k+1}^{*}-y_{k+1}^{*}, x-x_{k+1}\right\rangle \leq \frac{\gamma+M}{2 r} V\left(J\left(x_{k+1}\right), x\right), \quad \forall x \in C .
$$

On the other hand, by the fact that $y_{k+1}^{*} \in \partial^{\pi} F\left(x_{k+1}, \cdot\right)\left(x_{k+1}\right)$ and $F\left(x_{k+1}, \cdot\right)$ is $V$-uniformly prox-regular over $C$ with ratio $r^{\prime}$ we have

$$
\begin{aligned}
& \left\langle y_{k+1}^{*}, x-x_{k+1}\right\rangle \\
& \leq \frac{1}{2 r^{\prime}} V\left(J\left(x_{k+1}\right), x\right)+F\left(x_{k+1}, x\right) \\
& \quad-F\left(x_{k+1}, x_{k+1}\right) \quad \forall x \in C .
\end{aligned}
$$

Combining (37) and the last inequality we obtain

$$
\begin{aligned}
& \left\langle x_{k+1}^{*}, x-x_{k+1}\right\rangle \\
& \quad \leq F\left(x_{k+1}, x\right)+\left(\frac{\gamma+M}{2 r}+\frac{1}{2 r^{\prime}}\right) V\left(J\left(x_{k+1}\right), x\right) \quad \forall x \in C,
\end{aligned}
$$

which ensures that

$$
\begin{aligned}
& \left\langle x_{k+1}^{*}, x-x_{k+1}\right\rangle \\
& \quad \leq F\left(x_{k+1}, x\right)+\left(\frac{\gamma+M+1}{2 \bar{r}}\right) V\left(J\left(x_{k+1}\right), x\right) \quad \forall x \in C .
\end{aligned}
$$

This completes the proof of the proposition.

Now, we are ready to state and prove our first main theorem of this paper.

Theorem 12. Let $q>1$ and $X$ be a q-uniformly smooth Banach space. Let $C$ be a closed nonempty subset of $X$ and let $F: C \times C \rightarrow \mathbb{R}$ be a bifunction. Let $\left\{x_{k}\right\}_{k}$ be a sequence generated by (GSP1). Assume that

(1) $C$ is $V$-uniformly prox-regular with some ratio $r \in$ $(0, \infty]$; 
(2) $C$ is ball compact; that is, $C \cap M B$ is compact for any $M>0$;

(3) the solution set of $(G E P 1(C, \rho, F))$ is nonempty;

(4) $F$ is $W$-strongly monotone over $C$, that is,

$$
F(x, y)+F(y, x) \leq-\sigma W(x, y), \quad \forall x, y \in C,
$$

for some $\sigma \geq 0$;

(5) $F$ is upper semicontinuous with respect to the first variable over $C$, that is,

$$
\limsup _{x^{\prime} \rightarrow x} F\left(x^{\prime}, y\right) \leq F(x, y) \quad \forall x, y \in C ;
$$

(6) $F\left(x_{k+1}, \cdot\right)$ is $V$-uniformly prox-regular over $C$ with ratio $r^{\prime} \in(0,+\infty]$;

(7) there exists $\lambda>0$ such that $\lambda_{k} \geq \lambda$ for all $k$;

(8) the positive number $\rho \geq 0$ satisfies $(\gamma+M+$ 1) $/ 2 \min \left\{r, r^{\prime}\right\} \leq \rho \leq \sigma / 3$.

Then, there exists subsequence of $\left\{x_{k}\right\}$ that converges to $\tilde{x} \in C$ which solves $(G E P 1(C, \rho, F))$.

Proof. Let $\bar{x} \in C$ be a solution of $(G E P 1(C, \rho, F))$. Then

$$
F(\bar{x}, x) \geq-\rho V(J(\bar{x}), x), \quad \forall x \in C .
$$

By the $W$-strong monotonicity of $F$ over $C$ we have

$$
F(x, \bar{x})+F(\bar{x}, x) \leq-\sigma W(x, \bar{x}), \quad \forall x \in C .
$$

By setting $x=x_{k+1}$ in these two inequalities we get

$$
\begin{gathered}
F\left(x_{k+1}, \bar{x}\right)+F\left(\bar{x}, x_{k+1}\right) \leq-\sigma W\left(x_{k+1}, \bar{x}\right), \\
-F\left(\bar{x}, x_{k+1}\right) \leq \rho V\left(J(\bar{x}), x_{k+1}\right) .
\end{gathered}
$$

Combining these two inequalities we obtain

$$
\begin{aligned}
F\left(x_{k+1}, \bar{x}\right) & \leq \rho V\left(J(\bar{x}), x_{k+1}\right)-\sigma W\left(x_{k+1}, \bar{x}\right) \\
& \leq(2 \rho-\sigma) W\left(x_{k+1}, \bar{x}\right) .
\end{aligned}
$$

Using the 8th assumption of the theorem we have $2 \rho-\sigma \leq-\rho$ and hence

$$
F\left(x_{k+1}, \bar{x}\right) \leq-\rho W\left(x_{k+1}, \bar{x}\right) .
$$

This combined with Proposition 11 gives

$$
\begin{aligned}
& \left\langle x_{k+1}^{*}, \bar{x}-x_{k+1}\right\rangle \\
& \quad \leq\left(\frac{\gamma+M+1}{2 \bar{r}}\right) V\left(J(\bar{x}), x_{k+1}\right)-\rho W\left(x_{k+1}, \bar{x}\right) \\
& \quad \leq\left(\frac{\gamma+M+1}{\bar{r}}-\rho\right) W\left(x_{k+1}, \bar{x}\right),
\end{aligned}
$$

with $\bar{r}:=\min \left\{r, r^{\prime}\right\}$ and $x_{k+1}^{*}:=\lambda_{k}^{-1}\left[J\left(x_{k}\right)-J\left(x_{k+1}\right)\right]$. Therefore,

$$
\begin{aligned}
& \left\langle J\left(x_{k}\right)-J\left(x_{k+1}\right), \bar{x}-x_{k+1}\right\rangle \\
& \quad \leq \lambda_{k}\left(\frac{\gamma+M+1}{\bar{r}}-\rho\right) W\left(x_{k+1}, \bar{x}\right) .
\end{aligned}
$$

Define now the auxiliary real sequence $\phi_{k}=(1 / 2) V\left(J\left(x_{k}\right), \bar{x}\right)$. It is direct to check that

$$
\begin{aligned}
& 2\left[\phi_{k+1}-\phi_{k}\right]+V\left(J\left(x_{k}\right), x_{k+1}\right) \\
& \quad=2\left\langle J\left(x_{k}\right)-J\left(x_{k+1}\right), \bar{x}-x_{k+1}\right\rangle .
\end{aligned}
$$

Indeed,

$$
\begin{aligned}
2\left[\phi_{k+1}-\phi_{k}\right] & \\
= & V\left(J\left(x_{k+1}\right), \bar{x}\right)-V\left(J\left(x_{k}\right), \bar{x}\right) \\
= & {\left[\left\|J\left(x_{k+1}\right)\right\|^{2}-2\left\langle J\left(x_{k+1}\right), \bar{x}\right\rangle+\|\bar{x}\|^{2}\right] } \\
& -\left[\left\|J\left(x_{k}\right)\right\|^{2}-2\left\langle J\left(x_{k}\right), \bar{x}\right\rangle+\|\bar{x}\|^{2}\right] \\
= & \left\|J\left(x_{k+1}\right)\right\|^{2}+2\left\langle J\left(x_{k}\right)-J\left(x_{k+1}\right), \bar{x}\right\rangle-\left\|J\left(x_{k}\right)\right\|^{2} \\
= & 2\left\langle J\left(x_{k}\right)-J\left(x_{k+1}\right), \bar{x}\right\rangle-\left\|J\left(x_{k+1}\right)\right\|^{2} \\
& -\left\|J\left(x_{k}\right)\right\|^{2}+2\left\langle J\left(x_{k+1}\right), x_{k+1}\right\rangle \\
= & 2\left\langle J\left(x_{k}\right)-J\left(x_{k+1}\right), \bar{x}\right\rangle-V\left(J\left(x_{k}\right), x_{k+1}\right) \\
& -2\left\langle J\left(x_{k}\right), x_{k+1}\right\rangle+2\left\langle J\left(x_{k+1}\right), x_{k+1}\right\rangle \\
= & 2\left\langle J\left(x_{k}\right)-J\left(x_{k+1}\right), \bar{x}\right\rangle-V\left(J\left(x_{k}\right), x_{k+1}\right) \\
& -2\left\langle J\left(x_{k}\right)-J\left(x_{k+1}\right), x_{k+1}\right\rangle \\
= & 2\left\langle J\left(x_{k}\right)-J\left(x_{k+1}\right), \bar{x}-x_{k+1}\right\rangle-V\left(J\left(x_{k}\right), x_{k+1}\right) .
\end{aligned}
$$

It follows that

$$
\phi_{k+1}-\phi_{k} \leq\left\langle J\left(x_{k}\right)-J\left(x_{k+1}\right), \bar{x}-x_{k+1}\right\rangle,
$$

which ensures with (49) that

$$
\phi_{k+1}-\phi_{k} \leq \lambda_{k}\left(\frac{\gamma+M+1}{\bar{r}}-\rho\right) W\left(x_{k+1}, \bar{x}\right) .
$$

Using the assumption $\rho \geq(\gamma+M+1) / \bar{r}$ yields

$$
\phi_{k+1} \leq \phi_{k}
$$

Therefore, the sequence $\left\{\phi_{k}\right\}$ is a nonincreasing nonnegative sequence and so it is convergent to some limit and so it is bounded by some positive number $\alpha>0$. Thus, by the property (ii) of $V$ recalled in Section 1 we obtain

$$
\left(\|\bar{x}\|-\left\|x_{k}\right\|\right)^{2} \leq V\left(J\left(x_{k}\right), \bar{x}\right)=2 \phi_{k} \leq 2 \alpha,
$$

and so

$$
\left\|x_{k}\right\| \leq\|\bar{x}\|+\sqrt{2 \alpha}
$$

that is, $\left\{x_{k}\right\}$ is bounded and so by the $q^{\prime}$-uniform convexity of $X^{*}$ we have

$$
\left\|J\left(x_{k+1}\right)-J\left(x_{k}\right)\right\|^{q^{\prime}} \leq \eta V\left(J\left(x_{k}\right), x_{k+1}\right),
$$


for some $\eta>0$ depending on $\alpha$ and on the space $X^{*}$. Here $q^{\prime}=q /(q-1)$, where $q$ is the modulus of smoothness of $X$. Using now (49) and (50) and the assumption $\rho \geq(\gamma+M+1) / \bar{r}$ we obtain

$$
\frac{1}{2} V\left(J\left(x_{k}\right), x_{k+1}\right) \leq \phi_{k}-\phi_{k+1} .
$$

Therefore, it follows from the 7 th assumption of the theorem that

$$
\begin{aligned}
\left\|x_{k+1}^{*}\right\|^{q^{\prime}} & =\lambda_{k}^{-q^{\prime}}\left\|J\left(x_{k+1}\right)-J\left(x_{k}\right)\right\|^{q^{\prime}} \\
& \leq \lambda^{-q^{\prime}}\left\|J\left(x_{k+1}\right)-J\left(x_{k}\right)\right\|^{q^{\prime}} \\
& \leq \lambda^{-q^{\prime}} \eta V\left(J\left(x_{k}\right), x_{k+1}\right) \\
& \leq \frac{2 \eta}{\lambda q^{\prime}}\left[\phi_{k}-\phi_{k+1}\right] \longrightarrow 0 \text { as } k \longrightarrow \infty,
\end{aligned}
$$

which ensures that $\lim _{k \rightarrow \infty} x_{k+1}^{*}=0$. On the other hand, since $\left\{x_{k}\right\}$ is bounded in $C$ and $C$ is ball compact then there exists a subsequence $\left\{x_{k_{n}}\right\}$ which converges to some limit $\tilde{x} \in C$. Note that by Proposition 11 this subsequence satisfies

$$
\begin{aligned}
& \left\langle x_{k_{n}+1}^{*}, x-x_{k_{n}+1}\right\rangle \\
& \leq F\left(x_{k_{n}+1}, x\right) \\
& \quad+\left(\frac{\gamma+M+1}{2 \bar{r}}\right) V\left(J\left(x_{k_{n}+1}\right), x\right), \quad \forall n, \forall x \in C .
\end{aligned}
$$

Thus, by letting $n \rightarrow \infty$ in inequality (60) and by taking into account the upper semicontinuity of $F$ and the continuity of $V$ and $J$, we obtain

$$
0 \leq F(\tilde{x}, x)+\left(\frac{\gamma+M+1}{2 \bar{r}}\right) V(J(\tilde{x}), x), \quad \forall x \in C .
$$

Therefore, the assumption $\rho \geq(\gamma+M+1) / 2 \bar{r}$ concludes

$$
F(\tilde{x}, x)+\rho V(J(\tilde{x}), x) \geq 0 \quad \forall x \in C,
$$

which ensures that the limit $\tilde{x}$ is a solution of $(G E P 1(C, \rho, F))$.

As a direct consequence of the previous theorem we have the following convex version which is new according to our modest knowledge.

Theorem 13. Let $q>1$ and $X$ be a q-uniformly smooth Banach space. Let $C$ be a closed convex nonempty subset of $X$ and let $F: C \times C \rightarrow \mathbb{R}$ be a convex bifunction. Let $\left\{x_{k}\right\}_{k}$ be a sequence generated by (SP1). Assume that

(1) $C$ is ball compact; that is, $C \cap M B$ is compact for any $M>0$;

(2) the solution set of $(E P(C, F))$ is nonempty;

(3) $F$ is monotone over $C$; that is,

$$
F(x, y)+F(y, x) \leq 0, \quad \forall x, y \in C ;
$$

(4) $F$ is upper semicontinuous with respect to the first variable over $C$;

(5) there exists $\lambda>0$ such that $\lambda_{k} \geq \lambda$ for all $k$.

Then, there exists subsequence of $\left\{x_{k}\right\}$ that converges to $\tilde{x} \in C$ which solves $(E P(C, F))$.

Proof. It follows directly from the previous theorem with the constants $\rho=\sigma=0$ and $r=r^{\prime}=\infty$.

Now, we are going to prove a similar result for the second generalized equilibrium problem $(G E P 2(C, \rho, F))$. To do that we need a different and more restrictive concept of monotonicity that we define as follows: a bifunction $F$ is said to be $W$-relaxed strongly monotone with respect to $\left(\sigma_{1}, \sigma_{2}\right)$ for some $\sigma_{1} \geq 0$ and $\sigma_{2} \geq 0$ provided that

$$
\begin{array}{r}
F(x, y)+F(y, z) \leq-\sigma_{1} W(x, y)-\sigma_{2} W(y, z) \\
\forall x, y, z \in C .
\end{array}
$$

Observe that any $W$-relaxed strong monotone with respect to $\left(\sigma_{1}, \sigma_{2}\right)$ is $W$-strongly monotone with respect to $\sigma=\sigma_{1}+$ $\sigma_{2}$. As a simple example of $W$-relaxed strongly monotone bifunction we can take $F: X \times X \rightarrow \mathbb{R}$ defined by $F(x, y)=$ $W(x, y) /\left(\|x\|^{2}+3\right)-W(x, y)$. This bifunction is $W$-relaxed strongly monotone with respect to $(2 / 3,2 / 3)$. The proof of the following proposition follows the same lines of the proof of Proposition 11. So its proof is omitted. It is needed in the proof of Theorem 15.

Proposition 14. Let $X$ be a reflexive smooth Banach space and let $r, r^{\prime} \in(0,+\infty]$ and $\bar{r}:=\min \left\{r, r^{\prime}\right\}$. If $C$ is $V$ uniformly prox-regular with ratio $r \in(0, \infty]$ and $F\left(x_{k}, \cdot\right)$ is $\gamma$-Lipschitz and $V$-uniformly prox-regular over $C$ with ratio $r^{\prime}$, then the sequence $\left\{x_{k}\right\}_{k}$ generated by (GSP2) satisfies the following inequality:

$$
\begin{aligned}
& \lambda_{k}^{-1}\left\langle J\left(x_{k+1}\right)-J\left(x_{k}\right), x-x_{k}\right\rangle \\
& \quad \leq F\left(x_{k}, x\right)+\left(\frac{\gamma+M+1}{2 \bar{r}}\right) V\left(J\left(x_{k}\right), x\right), \quad \forall x \in C .
\end{aligned}
$$

Now, we prove our next main theorem concerning $(G E P 2(C, \rho, F))$.

Theorem 15. Let $q>1$ and $X$ be a q-uniformly smooth Banach space. Assume that Assumptions 1, 2, 3, 5, and 6 of Theorem 12 are satisfied and assume that $F$ is $W$-relaxed strongly monotone with respect to some $\left(\sigma_{1}, \sigma_{2}\right)$. If the constants $\sigma_{1}, \sigma_{2}, \gamma, \bar{r}, \rho$, and $\lambda$ satisfy the inequalities

$$
\begin{gathered}
\sigma_{2}>2 \rho+\frac{1}{\lambda}, \quad \rho \geq \frac{\gamma+M+1}{2 \bar{r}}, \\
\sigma_{1}+\sigma_{2} \geq \frac{\gamma+M+1}{\bar{r}}+\rho,
\end{gathered}
$$

then there exists a subsequence of the sequence $\left\{x_{k}\right\}$ generated by (GSP2) which converges to some solution of $(G E P 2(C, \rho, F))$. 
Proof. Let $\bar{x} \in C$ be a solution of $(G E P 2(C, \rho, F))$. By setting $x=x_{k+1}$ in $(G E P 2(C, \rho, F))$ we get

$$
-F\left(\bar{x}, x_{k+1}\right) \leq \rho V\left(J\left(x_{k+1}\right), \bar{x}\right)
$$

and by taking $x=\bar{x}$ in the conclusion of Proposition 14 we obtain

$$
\begin{aligned}
-F\left(x_{k}, \bar{x}\right) \leq & \lambda_{k}^{-1}\left\langle J\left(x_{k}\right)-J\left(x_{k+1}\right), \bar{x}-x_{k}\right\rangle \\
& +\alpha V\left(J\left(x_{k}\right), \bar{x}\right),
\end{aligned}
$$

with $\alpha:=(\gamma+M+1) / 2 \bar{r}$. The last two inequalities yield

$$
\begin{aligned}
- & {\left[F\left(x_{k}, \bar{x}\right)+F\left(\bar{x}, x_{k+1}\right)\right] } \\
& \leq \lambda_{k}^{-1}\left\langle J\left(x_{k}\right)-J\left(x_{k+1}\right), \bar{x}-x_{k}\right\rangle \\
& \quad+\rho V\left(J\left(x_{k+1}\right), \bar{x}\right)+\alpha V\left(J\left(x_{k}\right), \bar{x}\right) .
\end{aligned}
$$

Using the $W$-relaxed strong monotonicity of $F$ we write

$$
\begin{aligned}
- & {\left[F\left(x_{k}, \bar{x}\right)+F\left(\bar{x}, x_{k+1}\right)\right] } \\
& \geq \sigma_{1} W\left(x_{k}, \bar{x}\right)+\sigma_{2} W\left(\bar{x}, x_{k+1}\right) \\
& \geq \frac{\sigma_{1}}{2} V\left(J\left(x_{k}\right), \bar{x}\right)+\frac{\sigma_{2}}{2} V\left(J\left(x_{k+1}\right), \bar{x}\right) .
\end{aligned}
$$

Therefore, the two previous inequalities yield

$$
\begin{aligned}
\left(\frac{\sigma_{2}}{2}-\rho\right) V\left(J\left(x_{k+1}\right), \bar{x}\right) \\
\leq \lambda_{k}^{-1}\left\langle J\left(x_{k}\right)-J\left(x_{k+1}\right), \bar{x}-x_{k}\right\rangle \\
\quad+\left(\frac{\gamma+M+1}{2 \bar{r}}-\frac{\sigma_{1}}{2}\right) V\left(J\left(x_{k}\right), \bar{x}\right) .
\end{aligned}
$$

Observe that

$$
\begin{aligned}
& \left\langle J\left(x_{k}\right)-J\left(x_{k+1}\right), \bar{x}-x_{k}\right\rangle \\
& \quad=\frac{1}{2}\left[V\left(J\left(x_{k+1}\right), \bar{x}\right)-V\left(J\left(x_{k+1}\right), x_{k}\right)-V\left(J\left(x_{k}\right), \bar{x}\right)\right] .
\end{aligned}
$$

Hence,

$$
\begin{aligned}
& \left(\frac{\sigma_{2}}{2}-\rho-\frac{1}{2 \lambda_{k}}\right) V\left(J\left(x_{k+1}\right), \bar{x}\right) \\
& \quad \leq\left(\frac{\gamma+M+1}{2 \bar{r}}-\frac{\sigma_{1}}{2}-\frac{1}{2 \lambda_{k}}\right) V\left(J\left(x_{k}\right), \bar{x}\right) .
\end{aligned}
$$

We distinguish two cases.

Case 1. $\left((\gamma+M+1) / 2 \bar{r}-\sigma_{1} / 2-1 / 2 \lambda_{k}\right) \leq 0$. In this case (73) ensures that

$$
\left(\frac{\sigma_{2}}{2}-\rho-\frac{1}{2 \lambda_{k}}\right) V\left(J\left(x_{k+1}\right), \bar{x}\right) \leq 0
$$

and since $\left(\sigma_{2} / 2-\rho-1 / 2 \lambda_{k}\right)>0$ we obtain $V\left(J\left(x_{k+1}\right), \bar{x}\right)=$ 0 , for all $k$, that is, $x_{k+1}=\bar{x}$, for all $k$. This means that the sequence $\left\{x_{k}\right\}$ is constant and equals $\bar{x}$ and hence we are done.

Case 2. Consider $\left((\gamma+M+1) / 2 \bar{r}-\sigma_{1} / 2-1 / 2 \lambda_{k}\right)>0$. In this case we use our assumptions on the constants $\sigma_{1}, \sigma_{2}, r, r^{\prime}, \gamma$, $\rho$, and $\lambda$ to ensure that

$$
\frac{\sigma_{2}}{2}-\rho-\frac{1}{2 \lambda_{k}}>0, \quad \frac{(\gamma+M+1)-\bar{r} \sigma_{1}}{\bar{r} \sigma_{2}-2 \bar{r} \rho} \leq 1 .
$$

Thus, (73) can be rewritten as follows:

$$
\begin{aligned}
& V\left(J\left(x_{k+1}\right), \bar{x}\right) \\
& \quad \leq \frac{\left((\gamma+M+1) / 2 \bar{r}-\sigma_{1} / 2-1 / 2 \lambda_{k}\right)}{\left(\sigma_{2} / 2-\rho-1 / 2 \lambda_{k}\right)} V\left(J\left(x_{k}\right), \bar{x}\right) \\
& \quad \leq \xi V\left(J\left(x_{k}\right), \bar{x}\right) \leq V\left(J\left(x_{k}\right), \bar{x}\right),
\end{aligned}
$$

with $\xi:=\left((\gamma+M+1)-\bar{r} \sigma_{1}\right) /\left(\bar{r} \sigma_{2}-2 \bar{r} \rho\right) \leq 1$. The conclusion of the theorem follows the same lines as in the proof of Theorem 12.

Remark 16. An inspection of the proof of the previous theorem shows that in the case when $\xi<1$ the ball compactness of $C$ is not needed and that all the sequence $\left\{x_{k}\right\}$ converges to the solution $\bar{x}$, whenever the space $X$ is assumed to satisfy the $p$-uniform convexity instead of the $q$-uniform smoothness. Indeed, with the assumption $\xi<1$ (73) ensures $\lim _{k \rightarrow \infty} V\left(J\left(x_{k}\right), \bar{x}\right)=0$. This limit with the $p$ uniform convexity of the space $X$, and Lemma 1 ensure that $x_{k} \rightarrow \bar{x}$ and hence the proof is complete.

Corollary 17. Let $q>1, X$ be a q-uniformly smooth Banach space, $C$ be a closed convex nonempty subset of $X$, and let $F$ : $C \times C \rightarrow \mathbb{R}$ be a convex bifunction. Let $\left\{x_{k}\right\}_{k}$ be a sequence generated by (SP2). Assume that

(1) $C$ is ball compact; that is, $C \cap M B$ is compact for any $M>0$;

(2) the solution set of $(E P(C, F))$ is nonempty;

(3) $F$ satisfies for some $\sigma_{2}>0$

$F(x, y)+F(y, z) \leq-\sigma_{2} W(y, z), \quad \forall x, y, z \in C ;$

(4) $F$ is upper semicontinuous with respect to the first variable over $C$;

(5) there exists $\lambda>1 / \sigma_{2}$ such that $\lambda_{k} \geq \lambda$ for all $k$.

Then, there exists a subsequence of $\left\{x_{k}\right\}$ that converges to $\tilde{x} \in C$ which solves $(E P(C, F))$.

Proof. It follows directly from the previous theorem with the constants $\rho=\sigma_{1}=0$ and $r=r^{\prime}=\infty$.

It is a natural question to ask whether the additional assumption of $W$-relaxed strong monotonicity in Theorem 15 can be replaced by the one used in Theorem 12. The answer is given in the next theorem with a different generalized 
subproblem (GSP3) given by the following: select $x_{k+1} \in C$ such that

$$
\begin{aligned}
& \lambda_{k} F\left(x_{k}, x\right)+\lambda_{k} \alpha V\left(J\left(x_{k+1}\right), x\right) \\
& \quad+\left\langle J\left(x_{k+1}\right)-J\left(x_{k}\right), x-x_{k+1}\right\rangle \geq 0, \quad \forall x \in C .
\end{aligned}
$$

Theorem 18. Let $q>1$ and $X$ be a q-uniformly smooth Banach space. Assume that the assumptions 2-5 of Theorem 12 are satisfied and assume that the constants $\sigma, \alpha, \rho$, and $\lambda_{k}$ satisfy the inequalities

$$
\frac{\sigma}{2}-\rho \leq \alpha \leq \rho<\frac{\sigma}{2}, \quad 0<\lambda_{k}<\lambda:=\frac{1}{2 \alpha}, \quad \forall k .
$$

Then there exists a subsequence of the sequence $\left\{x_{k}\right\}$ generated by (GSP3) which converges to some solution of $(G E P 2(C, \rho, F))$.

Proof. Let $\bar{x} \in C$ be a solution of $(G E P 2(C, \rho, F))$. By setting $x=x_{k}$ in $(G E P 2(C, \rho, F))$ we get

$$
-F\left(\bar{x}, x_{k}\right) \leq \rho V\left(J\left(x_{k}\right), \bar{x}\right)
$$

and by taking $x=\bar{x}$ in (GSP3) we obtain

$$
\begin{aligned}
-F\left(x_{k}, \bar{x}\right) \leq & \lambda_{k}^{-1}\left\langle J\left(x_{k+1}\right)-J\left(x_{k}\right), \bar{x}-x_{k+1}\right\rangle \\
& +\alpha V\left(J\left(x_{k+1}\right), \bar{x}\right) .
\end{aligned}
$$

The last two inequalities yield

$$
\begin{aligned}
- & {\left[F\left(x_{k}, \bar{x}\right)+F\left(\bar{x}, x_{k}\right)\right] } \\
\leq & \lambda_{k}^{-1}\left\langle J\left(x_{k+1}\right)-J\left(x_{k}\right), \bar{x}-x_{k+1}\right\rangle \\
& +\rho V\left(J\left(x_{k}\right), \bar{x}\right)+\alpha V\left(J\left(x_{k+1}\right), \bar{x}\right) .
\end{aligned}
$$

Using the $W$-strong monotonicity of $F$ we write

$$
-\left[F\left(x_{k}, \bar{x}\right)+F\left(\bar{x}, x_{k}\right)\right] \geq \sigma W\left(x_{k}, \bar{x}\right) \geq \frac{\sigma}{2} V\left(J\left(x_{k}\right), \bar{x}\right) .
$$

Therefore, the two previous inequalities yield

$$
\begin{aligned}
& \lambda_{k}\left(\frac{\sigma}{2}-\rho\right) V\left(J\left(x_{k}\right), \bar{x}\right) \\
& \quad \leq\left\langle J\left(x_{k+1}\right)-J\left(x_{k}\right), \bar{x}-x_{k+1}\right\rangle+\alpha \lambda_{k} V\left(J\left(x_{k+1}\right), \bar{x}\right) .
\end{aligned}
$$

Observe that

$$
\begin{aligned}
& \left\langle J\left(x_{k+1}\right)-J\left(x_{k}\right), \bar{x}-x_{k+1}\right\rangle \\
& \quad=\frac{1}{2}\left[V\left(J\left(x_{k}\right), \bar{x}\right)-V\left(J\left(x_{k}\right), x_{k+1}\right)-V\left(J\left(x_{k+1}\right), \bar{x}\right)\right] .
\end{aligned}
$$

Hence,

$$
\begin{aligned}
& \lambda_{k}(\sigma-2 \rho) V\left(J\left(x_{k}\right), \bar{x}\right) \\
& \quad \leq V\left(J\left(x_{k}\right), \bar{x}\right)-V\left(J\left(x_{k+1}\right), \bar{x}\right)+2 \alpha \lambda_{k} V\left(J\left(x_{k+1}\right), \bar{x}\right),
\end{aligned}
$$

and so

$$
\begin{aligned}
& \left(1-2 \alpha \lambda_{k}\right) V\left(J\left(x_{k+1}\right), \bar{x}\right) \\
& \quad \leq\left(1-\lambda_{k}(\sigma-2 \rho)\right) V\left(J\left(x_{k}\right), \bar{x}\right) .
\end{aligned}
$$

Using our assumptions on the constants $\sigma, \alpha, \rho$, and $\lambda_{k}$, we have

$$
\left(1-2 \alpha \lambda_{k}\right)>0, \quad\left(1-\lambda_{k}(\sigma-2 \rho)\right)>0
$$

and hence we obtain

$$
V\left(J\left(x_{k+1}\right), \bar{x}\right) \leq \frac{\left(1-\lambda_{k}(\sigma-2 \rho)\right)}{\left(1-2 \alpha \lambda_{k}\right)} V\left(J\left(x_{k}\right), \bar{x}\right), \quad \forall k .
$$

Since by our assumptions we have $2 \alpha \geq \sigma-2 \rho$, then the function $t \mapsto(1-t(\sigma-2 \rho)) /(1-2 \alpha t)$ is nondecreasing and hence

$$
\frac{\left(1-\lambda_{k}(\sigma-2 \rho)\right)}{\left(1-2 \alpha \lambda_{k}\right)} \leq \frac{(1-\lambda(\sigma-2 \rho))}{(1-2 \alpha \lambda)} .
$$

Using the equality

$$
2 \alpha \geq \sigma-2 \rho
$$

once again yields $\xi:=(1-\lambda(\sigma-2 \rho)) /(1-2 \alpha \lambda)<1$. Therefore, (88) ensures

$$
V\left(J\left(x_{k+1}\right), \bar{x}\right) \leq \xi V\left(J\left(x_{k}\right), \bar{x}\right) \leq V\left(J\left(x_{k}\right), \bar{x}\right) \quad \forall k .
$$

Finally, the conclusion of the theorem follows the same lines as in the proof of Theorem 12 .

Remark 19. The same observation in Remark 16 holds for the previous theorem; that is, in the case when $\xi<1$ the ball compactness of $C$ is not needed and that the whole sequence $\left\{x_{k}\right\}$ converges to the solution $\bar{x}$, whenever the space $X$ is assumed to satisfy the $p$-uniform convexity instead of the $q$ uniform smoothness.

Corollary 20. Let $q>1, X$ be a q-uniformly smooth Banach space, let $C$ be a closed convex nonempty subset of $X$, and let $F: C \times C \rightarrow \mathbb{R}$ be a convex bifunction. Let $\left\{x_{k}\right\}_{k}$ be a sequence generated by (GSP3). Assume that

(1) $C$ is ball compact; that is, $C \cap M B$ is compact for any $M>0$;

(2) the solution set of $(E P(C, F))$ is nonempty;

(3) $F$ is monotone over $C$, that is,

$$
F(x, y)+F(y, x) \leq 0, \quad \forall x, y \in C ;
$$

(4) $F$ is upper semicontinuous with respect to the first variable over $C$;

(5) there exists $\lambda>0$ such that $\lambda_{k} \geq \lambda$ for all $k$.

Then, there exists subsequence of $\left\{x_{k}\right\}$ that converges to $\tilde{x} \in C$ which solves $(E P(C, F))$.

Proof. It follows directly from the previous theorem with the constants $\rho=\sigma=\alpha=0$ and $\lambda=\infty$. 


\section{Conflict of Interests}

The author declares that there is no conflict of interests regarding the publication of this paper.

\section{Acknowledgments}

The author would like to thank the referee for carefully reading the paper and for the well done report. This research was supported by the NSTIP strategic technologies program in Saudi Arabia, Award no. 11-MAT1916-02. Also, the author would like to thank Professor Hong-Kun $\mathrm{Xu}$ for the fruitful discussion on the subject of this research paper.

\section{References}

[1] M. Bounkhel and B. R. Al-Sinan, "An iterative method for nonconvex equilibrium problems," Journal of Inequalities in Pure and Applied Mathematics, vol. 7, no. 2, article 75, 8 pages, 2006.

[2] M. A. Noor, "Iterative schemes for nonconvex variational inequalities," Journal of Optimization Theory and Applications, vol. 121, no. 2, pp. 385-395, 2004.

[3] A. Moudafi, "Second-order differential proximal methods for equilibrium problems," Journal of Inequalities in Pure and Applied Mathematics, vol. 4, no. 1, article 15, 2003.

[4] M. A. Noor and K. I. Noor, "On equilibrium problems," Applied Mathematics E-Notes, vol. 4, pp. 125-132, 2004.

[5] M. A. Noor, K. I. Noor, and S. Zainab, "Some iterative methods for solving nonconvex bifunction equilibrium variational inequalities," Journal of Applied Mathematics, vol. 2012, Article ID 280451, 10 pages, 2012.

[6] M. Bounkhel, L. Tadj, and A. Hamdi, "Iterative schemes to solve nonconvex variational problems," Journal of Inequalities in Pure and Applied Mathematics, vol. 4, no. 1, article 14, 2003.

[7] G. Stampacchia, "Formes bilinéaires coercitives sur les ensembles convexes," Comptes Rendus de l'Académie des Sciences, vol. 258, pp. 4413-4416, 1964.

[8] R. Deville, G. Godefroy, and V. Zizler, Smoothness and Renormings in Banach Spaces, Pitman Monographs and Surveys in Pure and Applied Mathematics, Longman Scientific \& Technical, Harlow, UK, 1993.

[9] J. Diestel, Geometry of Banach Spaces, Selected Topics, vol. 485 of Lecture Notes in Mathematics, Springer, 1975.

[10] M. Bounkhel and R. Al-Yusof, "Proximal analysis in reflexive smooth Banach spaces," Nonlinear Analysis: Theory, Methods \& Applications, vol. 73, no. 7, pp. 1921-1939, 2010.

[11] F. H. Clarke, R. J. Stern, and P. R. Wolenski, "Proximal smoothness and the lower C2 property," Journal of Convex Analysis, vol. 2, no. 1, pp. 117-144, 1995.

[12] R. A. Poliquin, R. T. Rockafellar, and L. Thibault, "Local differentiability of distance functions," Transactions of the American Mathematical Society, vol. 352, no. 11, pp. 5231-5249, 2000.

[13] F. Bernard and L. Thibault, "Prox-regularity of functions and sets in Banach spaces," Set-Valued Analysis, vol. 12, no. 1-2, pp. 25-47, 2004. 


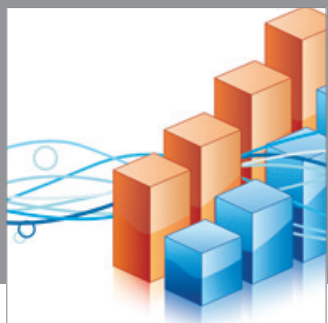

Advances in

Operations Research

mansans

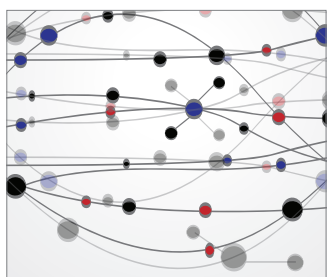

The Scientific World Journal
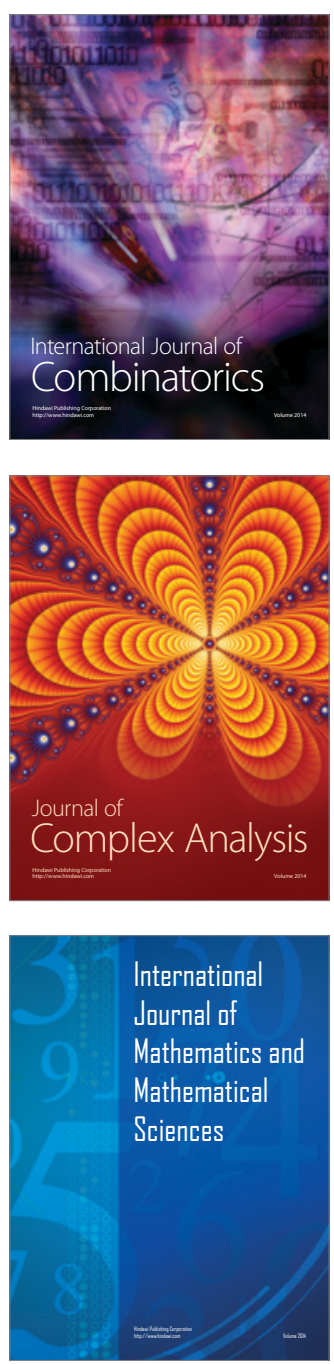
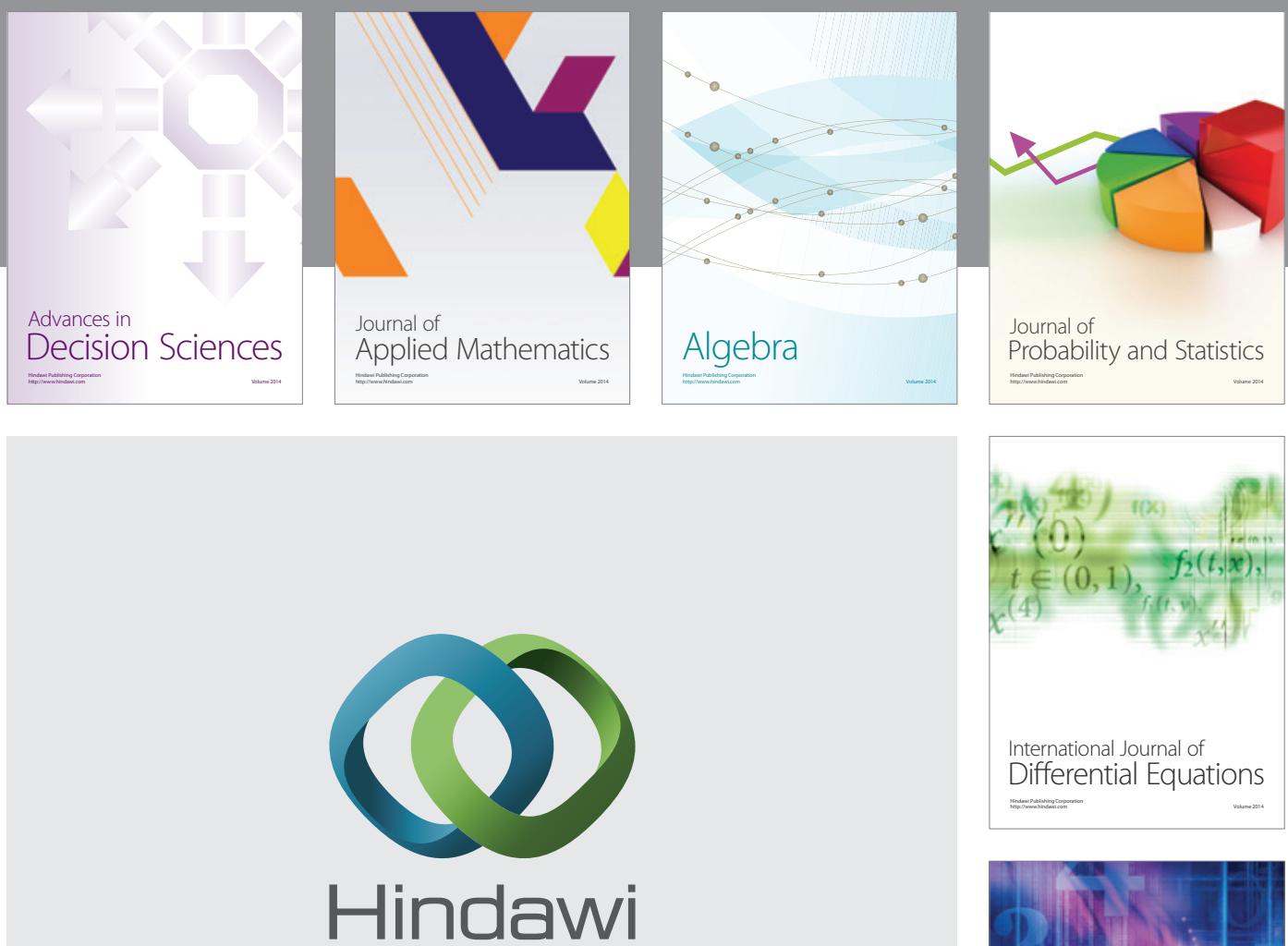

Submit your manuscripts at http://www.hindawi.com
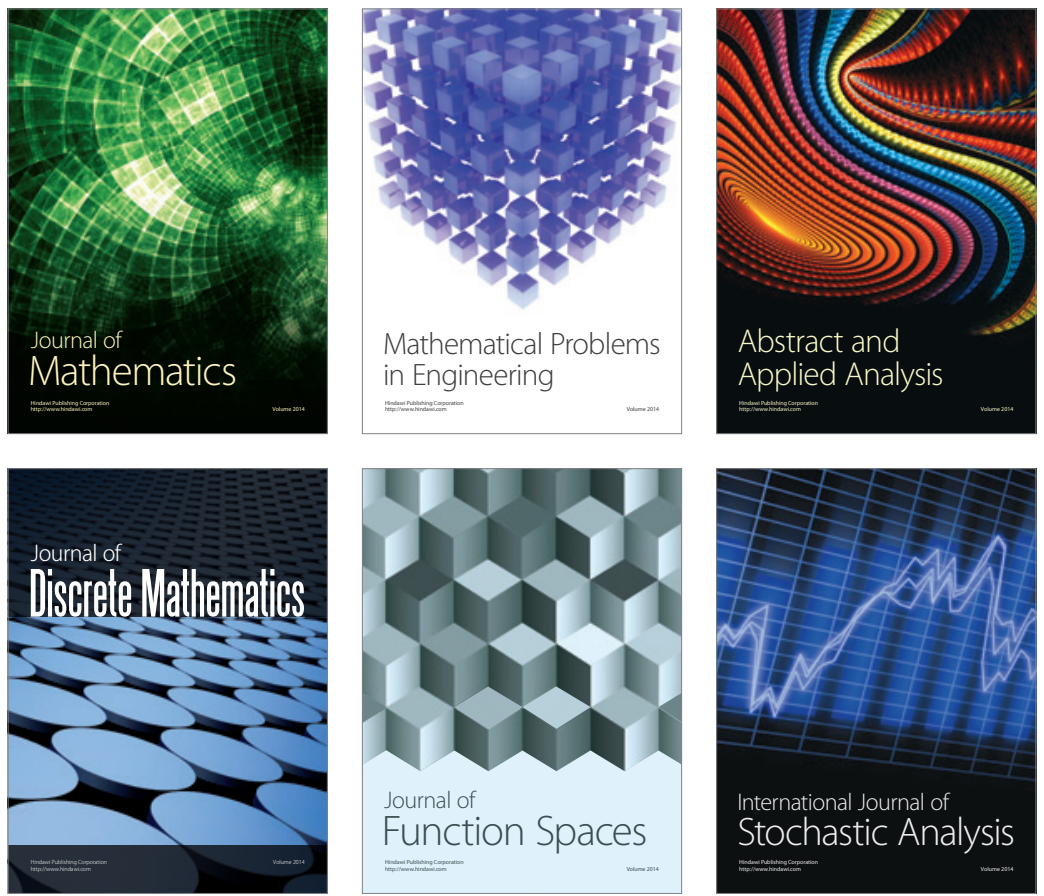

Journal of

Function Spaces

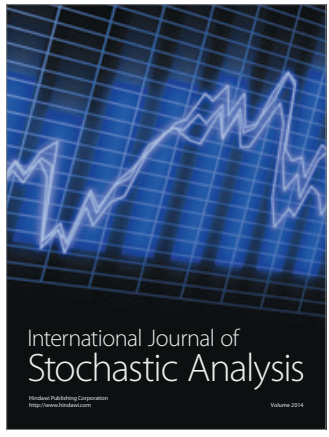

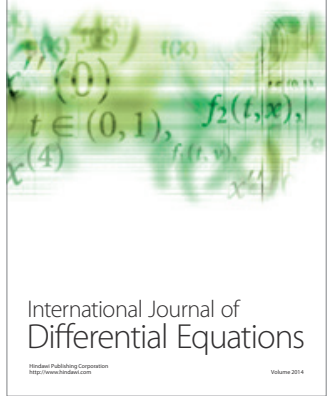
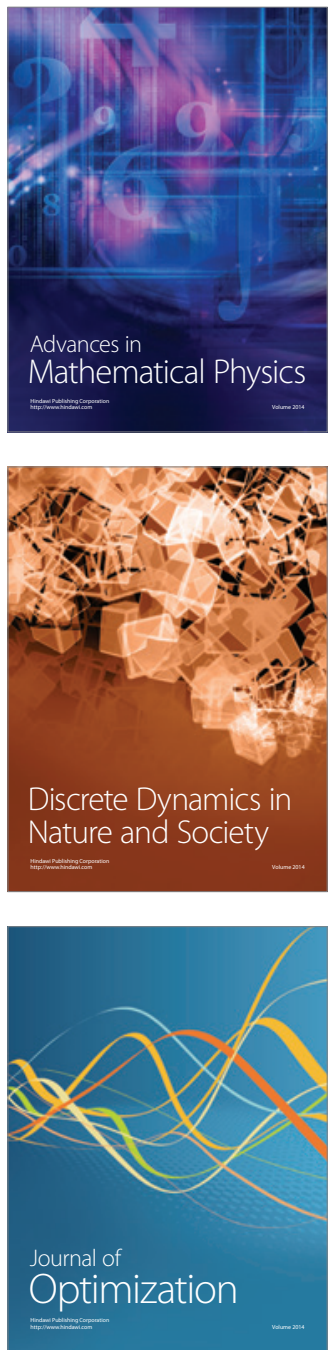